\title{
Numerical investigation of a fleet of self-propelled AUVs
}

\author{
Pareecha Rattanasiri ${ }^{1, *}$, Philip A. Wilson ${ }^{1}$, Alexander B. Phillips ${ }^{1}$ \\ Fluid-Structure Interaction Research Group, Faculty of Engineering and the Environment \\ University of Southampton, Southampton \\ United Kingdom SO17 1BJ
}

\begin{abstract}
This paper investigates the influence of the propeller on the drag of twin self-propelled AUVs, firstly, to examine the fleet performance for various propulsive conditions of leading and following AUV and, secondly, to study the parametric influence of transverse separations and longitudinal offsets on the fleet's drag. A series of CFD RANS-SST simulations have been performed at the Reynolds Number $3.2 \times 10^{6}$ with a commercial code ANSYS CFX 12.1. Mesh convergence is tested and validated with experimental and empirical results. The RANS-HO and RANS-UT propeller models are selected to estimate the time averaged thrust and torque of the propeller. The results show that the self-propelled vehicles experience an additional drag which is dominated by the thrust distribution of the propeller rather than torque. The drag of the following AUV is increased due to the upstream propeller, defined as a propeller race deduction. For the parametric studies, the results show that increasing the spacing results in a lower drag. The two sources of self-propelled drag increment are the viscous interaction and a direct result of proximity to the propeller race upstream. The result highlights the importance of considering both thrust deduction and any propeller race deductions when calculating the propulsive power consumption.
\end{abstract}

Keywords: co-operative AUVs, drag, RANS-SST, self-propelled model, body force model, propeller race deduction

\section{Introduction}

Autonomous Underwater Vehicles (AUVs) are self-propelled robots which perform missions without requiring external powering or an umbilical control. Current AUVs have the capability to perform missions such as: pipeline inspection (Labbe et al., 2004; Weiss et al., 2003), mine-sweeping (Edwards et al., 2004), and oceanographic exploration (Botelho et al., 2005; Martins et al., 2003). Proposed missions for the next generation of AUVs require both high quantity and quality temporal and spatial data. This enhanced performance may be achieved by either homogeneous or heterogeneous fleets of vehicles. By carrying different sensors, the surveying performance of multiple small vehicles may have a performance equal to or exceeding that of a single larger vehicle, with improved levels of robustness and redundancy for a specific task. This has led to various studies of manoeuvring and path planning of fleets (Aguiar et al., 2011; Bean et al., 2007; Burger et al., 2009; Cui et al., 2009, 2010; Reeder et al., 2004; Vanni, 2007).

Since the range of an AUV is dictated by its finite energy source, minimising the energy consumption is required to maximise endurance. For an individual AUV this may be achieved by obtaining the optimum hydrodynamic design e.g. hull, propeller and surface control (Bellingham and Willcox, 1996; Bradlev, 1992; Bradley et al., 2001; Dalton and Zedan, 1980; Huggins and Packwood, 1994; Jagadeesh and Murali, 2005, 2006; Jagadeesh et al., 2009; Kinsey, 1998; Parsons, 1972; Parsons et al., 1974; Phillips, 2009; Sarkar et al., 1997a; Stevenson et al., 2007). For a fleet of multiple AUVs, minimising the energy consumption may be targeted for both individuals and the entire fleet.

Recently, Rattanasiri et al. (2013) numerically investigated the influence of vehicle spacing among multiple towed spheroids on the individual drag and the combined fleet drag. The numerical results can be compared to the experimental studies of flow past bodies such as two circular cylinders, two slender bodies, in close proximity (Hoerner, 1965), a car with trailer and cars in convoy (Hucho and Ahmed, 1998), slipstreaming in cycling (Kyle, 1979), drafting in swimming (Silva et al., 2008), fish in schools or birds in flocks (Alexander, 2004;

\footnotetext{
${ }^{*}$ Corresponding author

Email addresses: pj506@soton.ac.uk (Pareecha Rattanasiri), philip.wilson@soton.ac.uk (Philip A. Wilson), abp@soton.ac.uk (Alexander B. Phillips)
} 
Andersson and Wallander, 2003; Hanrahan and Juanes, 2001; Partridge et al., 1983), dolphins in pods (Weihs, 2004; Weihs et al., 2007). Since the propeller's influence has been excluded from Rattanasiri et al. (2013) simulations, further investigation by modelling a propeller's influence on a fleet of AUVs will provide more information on the fleet propulsive energy budget.

Axial and tangential accelerations, applied to fluid by the action of the propeller, lead to significant variations in the flow field around the stern of the vessel and downstream, compared to a towed body. Firstly, the local pressure is reduced due to the flow acceleration, thus increasing the pressure drag acting on the body. Secondly, the objects placed downstream of the propeller experiences additional axial and tangential flow velocities in comparison to towed wake conditions. For an AUV to move forward at the design speed, the required thrust $\left(T_{S P}\right)$ must typically exceed its total towed drag $(R)$ (Burcher and Rydill, 1994):-

$$
R=(1-t) T_{S P}
$$

where $R$ is the drag of the bare hull and propeller and $t$ is defined as the thrust deduction which is a function of hull streamlining, propeller clearance and fullness (Burcher and Rydill, 1994).

As the numerical investigation of a fleet of self-propelled AUVs have never been studied (that we are awear of), the drag of twin self-propelled AUVs may be shown a decrease/increase by the generated wake upstream/downstream which lead to a saving/raising energy consumption. By neglecting the effect of appendages and other protrusion through the hull, these results lead to the underlying questions of:-

- does the impact of propeller provide the energy advantages/disadvantages to the fleet configuration?

- does a fleet configuration provide energy benefits for just an individual AUV or the whole fleet?

- what is the optimal configuration and optimal distances of the fleet?

The purpose of this paper is to provide guidance for operators on suitable spacing for multiple vehicles' missions. To achieve this aim, the two hydrodynamic processes of twin self-propelled AUVs: the body-to-body interference (or viscous interaction) and the increase of drag due to re-energised wake by a self-propelled vehicle must be numerically investigated.

\section{Theoretical approach}

To predict the hydrodynamic forces acting on an AUV's hull, a steady-state Reynolds Averaged Navier Stokes (RANS) simulation has proved to provide reasonably accurate results when compared against the experimental results (Jagadeesh et al., 2009; Karim et al., 2009; Phillips et al,, 2008, 2007, 2010c; Sarkar et al., 1997b). However, to obtain high fidelity result needs an appropriate mesh resolution to capture the effect of the boundary layer, body-to-body interaction and the wake behind the body.

The flow around a rotating propeller is a complex transient flow, high mesh resolution is required around the blade in order to resolve the flow features and small time steps are required to capture the transient flow behaviour. The problem considered in this paper does not concentrate on the propeller, but rather on a representative model of the velocity field downstream of the propeller. Therefore, this work utilises several body force propeller models which have been proposed for self-propelled ship simulations (Hough and Ordwav, 1965; Molland and Turnock, 1996; Paterson et al., 2003; Phillips et al., 2008, 2009a, 2010a; Stern et al., 1988; Turnock et al., 2008). The two considered models are;-

1. Uniform thrust distribution without torque, equivalent to an actuator disc applied over a finite thickness (RANS-UT propeller model)

2. Hough and Ordway model with prescribed radial distribution of thrust and torque (RANS-HO propeller model) (Hough and Ordway, 1965; Paterson et al., 2003; Stern et al., 1988)

When using these body force models, the geometry of the propeller is not explicitly represented. The effect of the propeller on the flow is modelled as distributed axial and tangential momentum source terms which induce axial and swirl accelerations in the fluid. Thus, with the defined magnitude and distribution of thrust and the torque on the propeller, the body force propeller can be implemented into the CFD-RANS code and simulates the self-propelled hulls. 


\subsection{Reynolds Averaged Navier Stokes (RANS)}

By assuming the flow is incompressible, the continuity equation becomes:-

$$
\frac{\overline{\partial U_{i}}}{\partial x_{i}}=0
$$

The momentum equation can be written as:-

$$
\rho\left(\frac{\partial \overline{U_{i}}}{\partial t}+\frac{\partial \overline{U_{i} U_{j}}}{\partial x_{j}}\right)=-\frac{\partial \bar{P}}{\partial x_{i}}+\frac{\partial}{\partial x_{j}}\left\{\mu\left(\frac{\partial \overline{U_{i}}}{\partial x_{j}}+\frac{\partial \overline{U_{j}}}{\partial x_{i}}\right)\right\}-\rho \frac{\partial \overline{u_{u}^{\prime} u_{j}^{\prime}}}{\partial x_{j}}+\bar{F}_{i}
$$

where the tensor $x_{i}$ represents Cartesian co-ordinates $(X, Y, Z)$ and $U_{i}$ are the Cartesian mean velocity components $\left(\overline{U_{x}}, \overline{U_{y}}, \overline{U_{z}}\right)$. The Reynolds stress tensor $\left(\overline{\rho u_{i}^{\prime} u_{j}^{\prime}}\right)$ is represented by the turbulence closure.

The RANS equations are implemented in the commercial CFD code ANSYS CFX (ANSYS, 2010). The governing equations are discretised using the finite volume method. A high-resolution advection scheme was applied for the results presented which varies between first- and second-order accuracy depending on spatial gradient. For a scalar quantity $\phi$ the advection scheme is written in the form of:-

$$
\phi_{i p}=\phi_{u p}+b \nabla \phi \cdot R_{u}
$$

where $\phi_{i p}$ is the value at the integration point, $\phi_{u p}$ the value at the upwind node and $R_{u}$ the vector from the upwind node to the integration point. The model reverts to first order when $b=0$ and is a second-order upwind biased scheme for $b=1$. The high-resolution scheme calculates $b$ using a similar approach to that of Brath and Jesperson (1989), which aims to maintain $b$ locally to be as close to one as possible without introducing local oscillations. Collocated (non-staggered) grids are used for all transport equations, and pressure velocity coupling is achieved using an interpolation scheme based on that proposed by Rhie and Chow (1982). Gradients are computed at integration points using tri-linear shape functions defined in ANSYS CFX (ANSYS, 2010). The linear set of equations that arise by applying the finite volume method to all elements in the domain are discrete conservation equations. The system of equations is solved using a coupled solver and a multigrid approach.

The shear stress transport (SST) turbulence closure model (Menter, 1994) which blends $k-\epsilon$ and $k-\omega$ was selected for this study. Previous investigations have shown that it is better able to replicate the flow around the ship and submarine hull forms than either $k-\epsilon$ or $k-\omega$ model, notably with a moderate computer accuracy (Larsson and Baba, 1996; Phillips et al., 2010b).

\subsection{Uniform thrust distribution model (RANS-UT)}

The simplest approach to modelling a propeller is to assume a uniform distribution of thrust over the propeller disc and neglect the torque. The axial and tangential momentum source terms are, respectively, given by;-

$$
\begin{aligned}
& F \bar{b}_{x}=\frac{T}{\Delta x \pi\left(R_{p}^{2}-R_{h}^{2}\right)} \\
& F \bar{b}_{\theta}=0
\end{aligned}
$$

Where $\Delta x$ is the thickness of the propeller subdomain, and $R_{h}$ and $R_{p}$ are the radii of the hub and propeller, respectively. The magnitude of $T$ must be provided by some other means, either experimental, based on the open-water data or from the numerical predictions.

\subsection{Hough and Ordway thrust and torque distribution model (RANS-HO)}

The radial distribution of thrust and torque is based on the Hough and Ordway (1965) circulation distribution, which has zero loading at the tip and root was shown to match Goldstein's optimum distribution (Goldstein, 1929). Coupling this distribution with a RANS simulation was proposed by Stern et al. (1988) and implemented by Paterson et al. (2003). The non-dimensional thrust distribution $\left(F \bar{b}_{x}^{\prime}\right)$ and the non-dimensional torque distribution $\left(F \bar{b}_{\theta}^{\prime}\right)$ are given by:-

$$
\begin{aligned}
& F \bar{b}_{x}^{\prime}=A_{x} r^{*} \sqrt{1-r^{*}} \\
& F \bar{b}_{\theta}^{\prime}=A_{\theta} \frac{r^{*} \sqrt{1-r^{*}}}{\left(1-Y_{h}\right) r^{*}+Y_{h}}
\end{aligned}
$$

where:

$$
A_{x}=\frac{C_{t h}}{\Delta x} \frac{105}{16\left(4+3 Y_{h}\right)\left(1-Y_{h}\right)}
$$




$$
\begin{aligned}
& C_{t h}=\frac{8 K_{T}}{\pi J^{2}}=\frac{2 T}{\rho V_{a}^{2} \pi R_{p}^{2}} \\
& A_{\theta}=\frac{K_{Q}}{\Delta x J^{2}} \frac{105}{\pi\left(4+3 Y_{h}\right)\left(1-Y_{h}\right)} \\
& J=V_{a} / n\left(2 R_{p}\right) \\
& V_{a}=\left(1-w_{t}\right) V
\end{aligned}
$$

where both $C_{t h}$ and $K_{T}$ are called the thrust coefficient with different form as shown in Euqation 10 , $J$ is the advance coefficient. $T$ is the thrust. $w_{t}$ is the wake fraction. $n$ is the revolutions per second. $V$ is the vehicle speed and $V_{a}$ is the advance speed. The momentum source terms are then applied to an annulus with finite thickness $(\Delta x)$ as shown in Figure 1 which is defined by:-

$$
Y_{h}=\frac{R_{h}}{R_{p}}, \quad Y=\frac{r_{p}}{R_{p}}, \quad r^{*}=\frac{Y-Y_{h}}{1-Y_{h}}
$$

where $r_{p}$ is the local propeller radius.

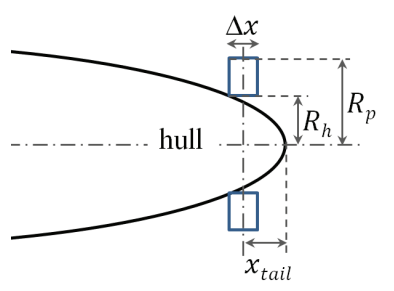

Figure 1: Body force propeller model

\section{Case study}

\subsection{Base experiment}

Molland and Utama (1997) performed a series of experiments on twin prolate spheroids to characterise the side-force and yawing moment interactions. Tests were carried out in the $7^{\prime} \times 5^{\prime}(2.20 \mathrm{~m} \times 1.57 \mathrm{~m})$ low speed wind tunnel at the University of Southampton. The overall length of each model was $1200 \mathrm{~mm}$ with maximum diameter of $200 \mathrm{~mm}$ and a surface area $\left(A_{w}\right)$ of $0.601 \mathrm{~m}^{2}$. The top spheroid was placed at the middle breadth and $1.07 \mathrm{~m}$ height from the floor. It was fitted to the overhead wind tunnel dynamometer for measuring the total drag and side-force. The lower spheroid (B2) was placed at the transverse separation $(S / L)$ of $0.27,0.37$ and 0.47 away from B1. The noses of both spheroids are aligned with zero longitudinal offset $(D / L=0)$ as shown in Figure 2 .

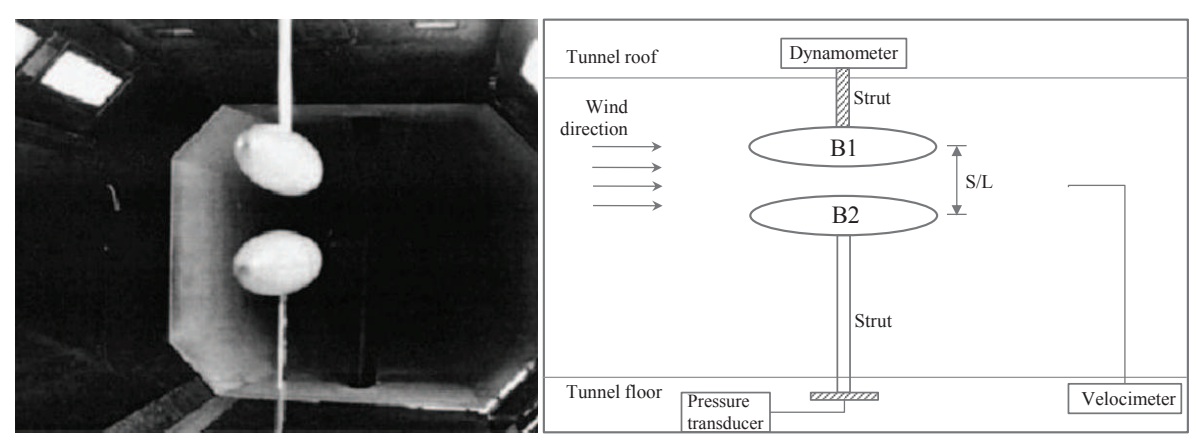

Figure 2: Set up of twin prolate spheroids in the $14 \mathrm{~m} \mathrm{long}, 7^{\prime} \times 5^{\prime}$ wind tunnel, figure adapted from (Molland and Utama, 1997) 


\subsection{Assumed propeller characteristics}

The propeller performance is scaled from Autosub3 (Phillips, 2009), the ratio of propeller diameter to maximum hull diameter is 0.8 . The $K_{T}$ and $K_{Q}$ of Autosub3 related to $J$ is predicted by the following equations (Phillips et al., 2009b):-

$$
\begin{aligned}
& K_{T}=0.5106 J^{3}-0.7700 J^{2}+0.2017 J+0.0529 \\
& 10 K_{Q}=0.3920 J^{3}-0.7499 J^{2}+0.3218 J+0.0032
\end{aligned}
$$

The ratio of finite length of the propeller disc and maximum diameter of the disc is suggested to be $\geq 0.1$ (Phillips et al., 2010a). The other parameters are in the Table1.

Table 1: Parameter of modelled propeller

\begin{tabular}{|c|c|c|c|c|}
\hline Parameters & $R_{p}(\mathrm{~m})$ & $R_{h}(\mathrm{~m})$ & $\Delta x(\mathrm{~m})$ & $x_{\text {tail }}(\mathrm{m})$ \\
\hline Setting & 0.080 & 0.0154 & 0.0154 & 0.007 \\
\hline
\end{tabular}

In this study, the propeller rpm is iteratively varied to determine the appropriate advance coefficient (Equation 12), thrust coefficient (Equation 15) and torque coefficient (Equation 16) to achieve a self-propulsion.

\subsection{Previous numerical study of towed vehicles}

Rattanasiri et al. (2013) simulated the experiment of Molland and Utama (1997) by considering the twin towed spheroids at various transverse separations. The results exhibited good correlation with the pressure distribution, the side-force coefficient, form factor and the drag. The numerical method and the mesh strategy in Rattanasiri et al. (2013) can therefore be used to achieve high fidelity results.

\subsection{Present study}

The action of the propeller is to re-energise the wake compared to a towed vessel. This propeller race will interact with any vehicles directly downstream impacting on their own drag. This study uses a body force approach to replicate the action of the propeller so the drag of a fleet of self-propelled vehicles can be investigated.

Initial simulations are performed for a single hull. Case S1 is the benchmark towed single hull. Cases S2 and S3 correspond to a self-propelled vehicle using the two body force propeller models RANS-UT and RANS-HO, respectively. Comparing S2 and S3, the important of considering the propeller's torque and resulting in swirling flow may be examined.

Subsequently, simulations are performed for a fleet of two vehicles. Cases F1 to F6 examine a fleet performance for various combinations of the towed and self-propelled vehicles using the two body force models at $S / L=0$ and $D / L=1.47$. Cases F1 to F3 are then extended to consider two self-propelled vehicles at a selection of longitudinal offset for $1.17 / L \leq 4.47$ (Figure 3a) and transverse separations for $0.17 / L \leq 0.47$ (Figure 3b).

Table 2: Propeller test case matrix to simulate twin self-propelled vehicles in $S / L=0$ and $D / L=1.47$ configuration at various propulsive conditions. Where the simple uniform thrust distribution is simulated by the RANS-UT propeller model and the thrust and torque propeller model is simulated by the RANS-HO propeller model

\begin{tabular}{|c|c|c|}
\hline Case & B1 & B2 \\
\hline S1 & Towed Hull & - \\
S2 & Thrust Propeller Model & - \\
S3 & Thrust and Torque Propeller Model & - \\
F1 & Towed Hull & Towed Hull \\
F2 & Thrust Propeller Model & Towed Hull \\
F3 & Thrust Propeller Model & Thrust Propeller Model \\
F4 & Thrust and Torque Propeller Model & Towed Hull \\
F5 & Thrust and Torque Propeller Model & Thrust Propeller Model \\
F6 & Thrust and Torque Propeller Model & Thrust and Torque Propeller Model \\
\hline
\end{tabular}




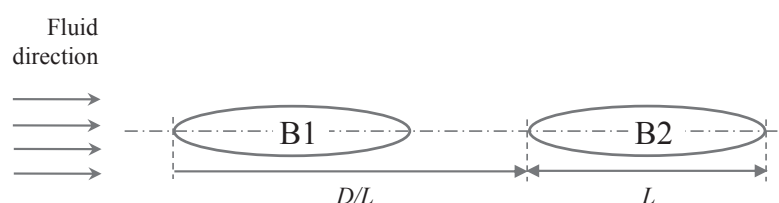

(a) Drafting configuration

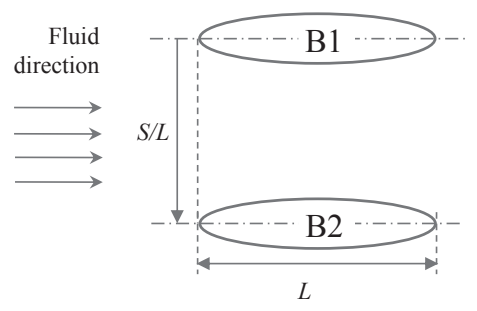

(b) Parallel configuration

Figure 3: A pair of prolate spheroids in fleet configurations

\subsection{Numerical settings}

To replicate the experiment of Molland and Utama (1997), the dimension of fluid domain is modelled at $1.4 L \times$ $12 L \times 1.8 L$. Free slip wall conditions (ANSYS, 2010) are used for the roof, floor and walls. The air inlet velocity $(V)$ is set at $40 \mathrm{~m} / \mathrm{s}$ related to a length Reynolds number $(\operatorname{Re}) 3.2 \times 10^{6}$ (For typical AUV, $10^{5}<\operatorname{Re}<10^{7}$ ) with the zero relative pressure outlet. The air density $\left(\rho_{\text {air }}\right)$ and the air kinematic viscosity $\left(v_{\text {air }}\right)$ at room temperature are $1.185 \mathrm{~kg} / \mathrm{m}^{3}$ and $1.545 \times 10^{-5} \mathrm{~m}^{2} / \mathrm{s}$, respectively. Both hulls are modelled by using no slip wall condition (ANSYS, 2010).

\subsection{Mesh strategy}

The model domain, boundary condition and mesh strategies used in this simulation are illustrated in Figure 4 The sample of meshes cut in the ZX plane and the YZ plane is shown in Figure 5a and 5b, respectively. A series of eleven meshes ranging from 1.2 to 22.7 million elements were made as an example cases, where the 1.2, 8.6 and 22.7 million element are designated as the coarse mesh, the medium mesh and the fine mesh, respectively. With a $2.0 \%$ difference of total drag between the medium mesh (case f) and the fine mesh (case j), the computational time of the medium mesh is approximately 8 hours which is 10 times quicker than that of fine mesh by using the 64-bit operating computational system, $2.53 \mathrm{GHz}, 2$ Core Processors with 12GB RAM. Thus the mesh parameters of global mesh size and the local refinement case $\mathrm{f}$ is selected as appropriate. The computational parameters are provided in Table 3

This numerical setting and the mesh strategy have proved to provide a good agreement between the twin towed bare hulls' drag of Rattanasiri et al. (2013) numerical simulation and the experimental results (Molland and Utama, 1997), the numerical results (Molland and Utama, 2002) and the empirical results (Hoerner, 1965). Thus by implementing a propeller model into the numerical twin towed hulls of Rattanasiri et al. (2013), the investigation of the impact of propeller to a fleet of self-propelled twin hulls can be performed. 


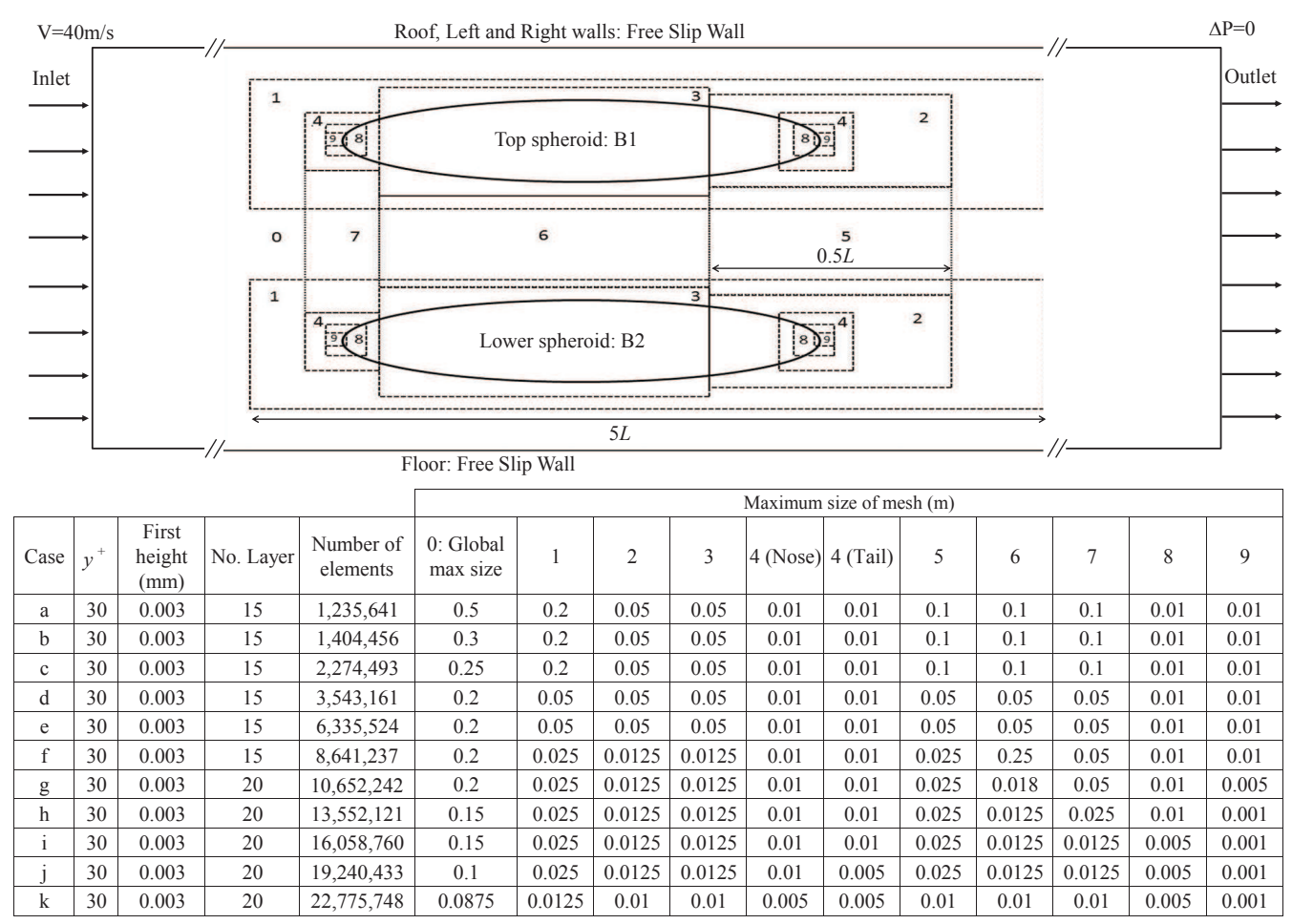

Figure 4: (Top) Boundary condition and mesh refinement around a pair of prolate spheroids (Bottom) Eleven mesh strategies

Table 3: Computational parameters

\begin{tabular}{|l|l|}
\hline Parameters & Setting \\
\hline Global mesh size & $0.20 \mathrm{~m}$ \\
Mesh type & $\begin{array}{l}\text { Unstructured with local } \\
\text { refinement around spheroids } \\
\text { and in wake regions }\end{array}$ \\
& 30 \\
$y^{+}$average & $8-15 \mathrm{M}$ with 15 prism layers \\
No. of elements & in the boundary layer \\
& Shear Stress Transport \\
Turbulence model & $1 \%$ \\
Inlet turbulent intensity & Automatic Wall Function (ANSYS, 2010) \\
Wall modelling & High Resolution (ANSYS, 2010) \\
Spatial discretisation & Auto Timescale (ANSYS, 2010) \\
Timescale control & RMS residual < 10 \\
Convergence criteria & Iridis 3 Linux Cluster \\
Computing & Parallel (12 partitions run on \\
Run type & $4 \times$ Dual core nodes, \\
& each with 2GB RAM) \\
Simulation Time & $2.0-2.5$ wall clock hours \\
\hline
\end{tabular}




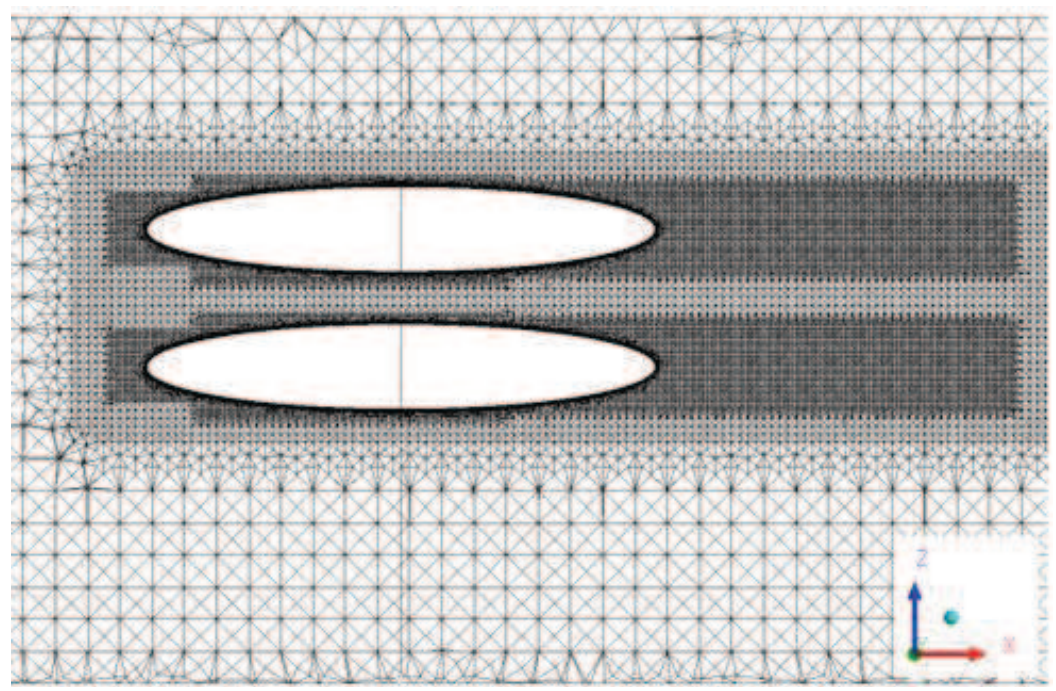

(a) ZX plane at the centre line at $Y=0$ with the fluid flow from left to right

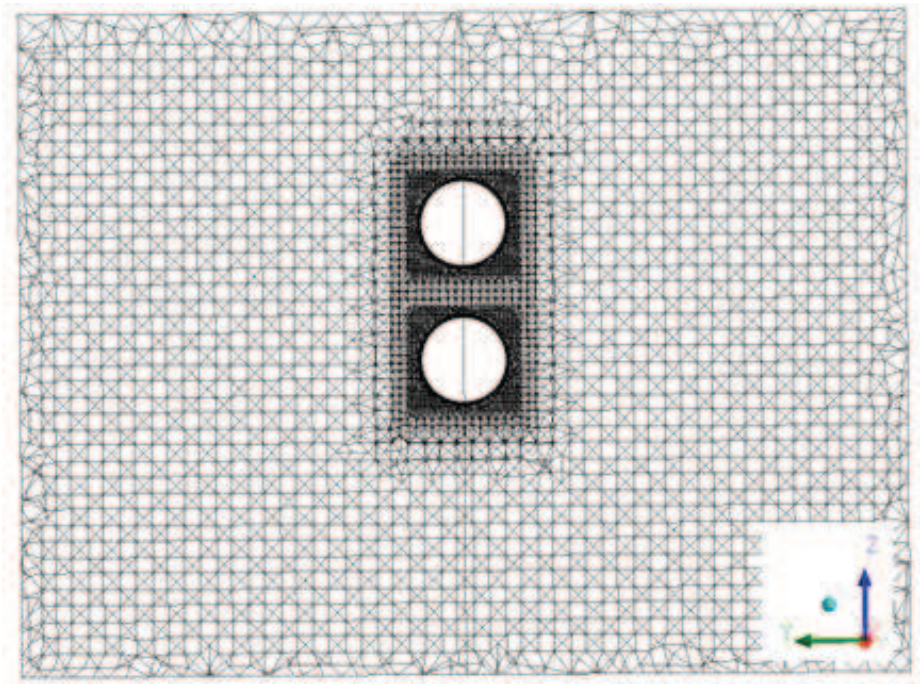

(b) YZ plane at $X=0.6 \mathrm{~m}$ from the noses

Figure 5: Mesh cut around a pair of spheroids for $S / L=0.27$ and $D / L=0$ 


\section{Results}

\subsection{Single vehicle}

Table 4 details the thrust deduction calculated for a single hull using the two body force models. The propeller revolution speed of each self-propelled AUV is iterated until the error between the self-propelled thrust $\left(T_{S P}\right)$ and the self-propelled drag $\left(T_{D}\right)$ are within $0.2 \%$. By assuming no swirl imparted to the flow $\left(K_{Q}=0\right)$, a uniform thrust distribution RANS-UT model predicted the thrust deduction, $t=0.13$. The more characteristic thrust and torque distributions of RAN-HO model gave $t=0.14$. The numerical predictions of thrust deduction $(t)$ are within the ranges suggested in Burcher's experiments (Burcher and Rydill, 1994).

Table 4: Thrust deduction and drag results of a single self-propelled AUV with two different propulsive conditions

\begin{tabular}{|c|c|c|c|c|c|}
\hline Body Force Model & $\begin{array}{c}\text { Towed } \\
\mathrm{drag}, \\
R(\mathrm{~N})\end{array}$ & $\begin{array}{c}\text { Self-propelled } \\
\text { thrust, } \\
T_{S P}(\mathrm{~N})\end{array}$ & $\begin{array}{c}\text { Self-propelled } \\
\mathrm{drag}, \\
T_{D}(\mathrm{~N})\end{array}$ & $\begin{array}{c}\text { \%error, } \\
\% \frac{T_{D}-T_{S}}{T_{S P}}\end{array}$ & $\begin{array}{c}\text { Thrust } \\
\text { deduction, } \\
t\end{array}$ \\
\hline S2: Thrust Propeller (RANS-UT) & 2.3014 & 2.6393 & 2.6426 & 0.17 & 0.13 \\
\hline S3: Thrust and Torque Propeller (RANS-HO) & 2.3014 & 2.6830 & 2.6874 & 0.12 & 0.14 \\
\hline
\end{tabular}

Table 5 shows a breakdown of the pressure and skin friction components of the drag for cases S1, S2 and S3. The two self-propelled cases (S2 \& S3) show an increament of 14.7\% and 16.6\% in the total self-propelled drag compared to the towed case (S1), respectively. The discrepency is driven by a change to the pressure drag. These cases demonstrate the importance of considering the self-propelled drag when assessing total energy budget of an AUV.

Table 5: Drag coefficient of a single towed hull and a single self-propelled AUV with two different propulsive conditions. Define the percent drag difference compare to case $\mathrm{S} 1$ as $\% \Delta_{t o}{ }_{1}=\frac{\operatorname{drag}_{i}-d r a g_{(S 1)}}{\operatorname{drag}_{(S 1)}}$, where $i$ is cases $\mathrm{S} 2$ and S3

\begin{tabular}{|c|c|c|c|c|c|c|}
\hline Cases & $\begin{array}{l}\text { Total drag } \\
\text { coef. } \\
C_{D} \times 10^{3} \\
\end{array}$ & $\% \Delta_{(S 1)}$ & $\begin{array}{c}\text { Skin friction } \\
\text { drag coef. } \\
C_{F} \times 10^{3}\end{array}$ & $\% \Delta_{(S 1)}$ & $\begin{array}{c}\text { Pressure } \\
\text { drag coef. } \\
C_{P x} \times 10^{3}\end{array}$ & $\% \Delta_{(S 1)}$ \\
\hline $\begin{array}{c}\text { S1: Towed hull } \\
\text { (No propeller model) }\end{array}$ & 4.053 & & 3.643 & & 0.347 & \\
\hline $\begin{array}{l}\text { S2: Simple Thrust Propeller } \\
\text { (RANS-UT) }\end{array}$ & 4.632 & 14.7 & 3.647 & 0.1 & 1.012 & 191.6 \\
\hline $\begin{array}{l}\text { S3: Thrust and Torque Propeller } \\
\text { (RANS-HO) }\end{array}$ & 4.709 & 16.6 & 3.648 & 0.1 & 1.089 & 213.8 \\
\hline
\end{tabular}

\subsection{Drafting twin vehicles at $D / L=1.47$}

The flow velocity past two drafting hulls $(S / L=0$ and $D / L=1.47)$ is demonstrated in Figure 6 . Three cases are shown:

- case F1 where both hulls are towed (dotted line)

- case F2 where the leader B1 is the self-propelled vehicle and the follower B2 is towed (dashed line)

- case F3 where both vehicles are self-propelled (continuous line)

From a uniform inflow $(D / L=-1.250)$, the boundary layer develops over $\mathrm{B} 1(0<D / L<1.0)$. At the propeller plane of $\mathrm{B} 1(D / L=0.994)$, a difference may be observed between cases $\mathrm{F} 1$ and $\mathrm{F} 2 \& \mathrm{~F} 3$. The advance velocity for $\mathrm{B} 1$ for the towed case $\mathrm{F} 1$ is $34.05 \mathrm{~m} / \mathrm{s}$ which corresponds to a mean fraction wake $\left(\bar{w}_{t}\right)$ of 0.148 (calculated by Equation 13). Cases F2 and F3 experience an increase in the axial momentum on the propeller plane 1. This axial increment increases with the slipstream contraction at $D / L=1.235$.

The inflow to $\mathrm{B} 2(D / L=1.470)$ differs for the towed $\mathrm{B} 1(\mathrm{~F} 1)$ where there is a wake deficit and the selfpropelled B1 (F2\&F3) where the propeller race has re-energised the wake, however, this is a non uniform inflow. The boundary layer then develops around B2 at $1.470<D / L<2.463$. At the propeller plane of B2 $(D / L=2.463)$, the mean wake fraction for $\mathrm{B} 2$ for case $\mathrm{F} 2$ is 0.143 . 
Downstream at $D / L=4.789$, the three cases show different behaviour where F1 exhibites the largest wake deficit, F3 the smallest. Case F1 exhibits a wake deficit equating to both vehicles' drag, F2 exhibits a wake deficit due to B2's drag and the re-energised wake of the propeller 1, and F3 exhibits a nearly completely uniform flow since both vehicles are self-propelled.
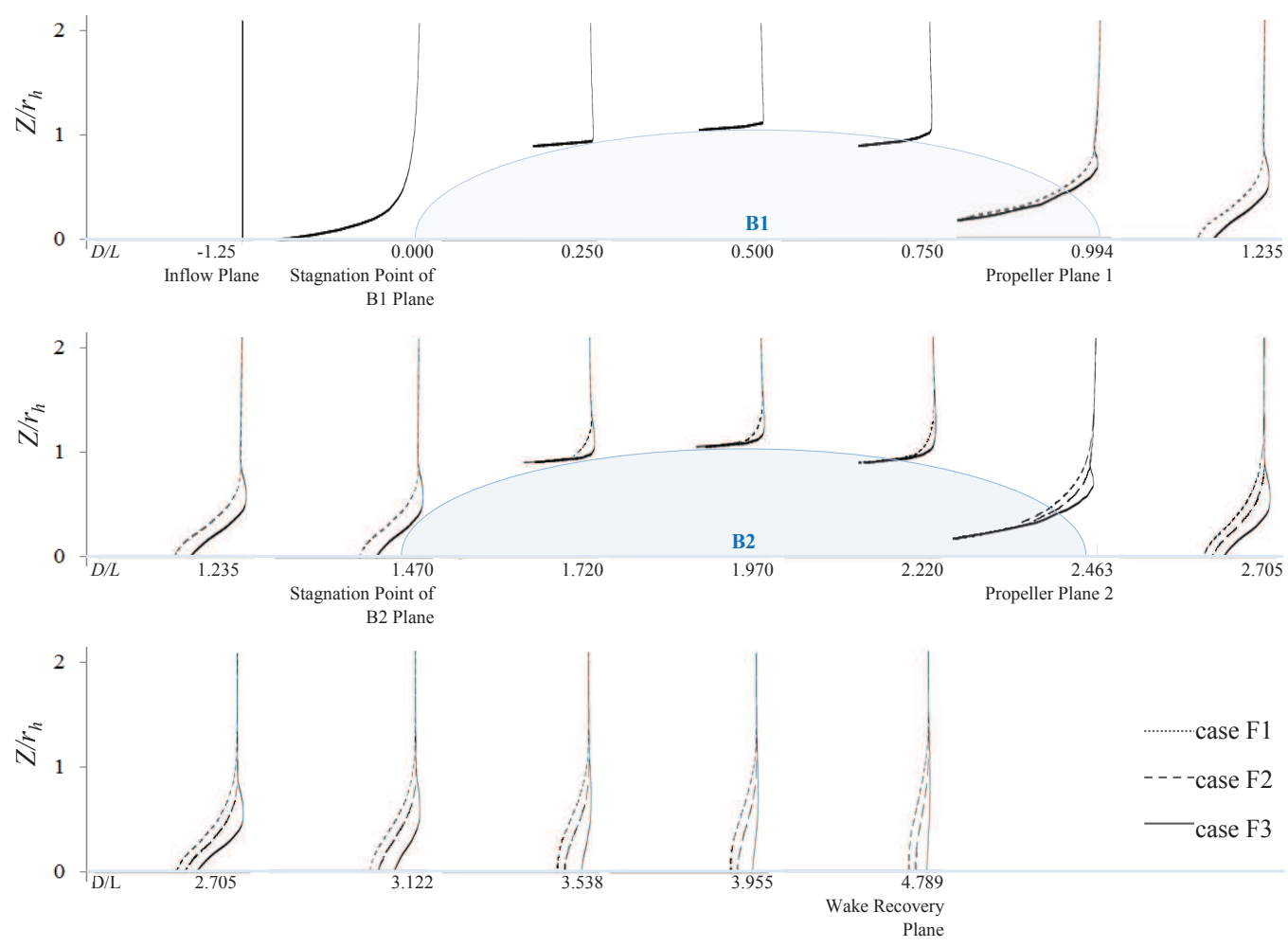

Figure 6: The velocity profile of the flow past two in-line hulls $(S / L=0, D / L=1.47)$. case F1 where both hulls are towed (dotted line), case F2 where the leader (B1) is the self-propelled vehicle and the follower (B2) is towed (dashed line), and a final case F3 where both vehicles are self-propelled (continuous line)

The influence of the propeller model on the self-propelled drag, the thrust deduction and the propeller race deduction are demonstrated in Figure 7 for the drafting $\mathrm{B} 1$ and $\mathrm{B} 2$ at $D / L=1.47$. The evaluated thrust deduction, $t$, of each case is within the empirical range of 0.13-0.14.

Starting with the twin towed case F1, the drag of B1 is reduced compared to a single towed vehicle. This is due to the bow stagnation of B2 aiding the pressure recovery at the stern of B1. For cases F2 to F6, B2 experiences higher drag than case F1 due to the propeller race at B1. This can be captured by using a propeller race deduction, $\gamma$. Thus the towed drag of the follower vehicle B2 (cases F2 and F4) may be calculated from;-

$$
R_{\mathrm{B} 2 \text { (towed } \mathrm{B} 1)}=(1-\gamma) R_{\mathrm{B} 2 \text { (self-propelled } \mathrm{B} 1)}
$$

Hence, for a self-propelled follower B2 of cases F3, F5 and F6;-

$$
R_{\mathrm{B} 2 \text { (towed B1) }}=(1-\gamma-t) T_{S P, \mathrm{~B} 2 \text { (self-propelled B1) }}
$$

Comparing the results from the RANS-UT and RANS-HO body force approaches, it is evident that the momentum source terms which represent propeller thrust are governing the predicted thrust deduction and propeller race deduction. 


\begin{tabular}{cl|l|l|l|l|l|} 
Compare case $i$ to case $j$ \\
\hline Case
\end{tabular}

Figure 7: Drag force results and the percentage drag differences in the drafting configuration at $D / L=1.47$, where $d r a g$ represent the towed drag $(R)$ for a towed case and the self-propelled thrust $\left(T_{S P}\right)$ for any propulsive conditions, $t$ is the thrust deduction and $\gamma$ is defined as the propeller race deduction.

\subsection{Twin vehicles at various drafting and parallel configurations}

From the previous results, the swirling effect of the propeller can be neglected and the simplified RANS-UT model can be selected for parametric studies into the effect of spacing between two hulls.

Figure 8 illustrates the influence of the longitudinal offset in the drafting configuration on the components of drag. Starting with case F1, at $D / L=1.17$, the total drag of $\mathrm{B} 1$ is increased by $8 \%$ whilst the total drag of $\mathrm{B} 2$ is reduced by $21 \%$ (Figure 8a). As the longitudinal offset increases, the drag of both towed hulls tends towards the free stream drag as the wake recovery occurs. This hydrodynamic behaviour is similar to cars in a convoy with a slipstream at $S / L>1.5$ (Hucho and Ahmed, 1998). This goes some-way to explain the energy benefit of cycling and swimming in the slipstream (Kyle, 1979; Silva et al., 2008). According to Figure 8b and 8c the discrepency of the total drag of both hulls is driven by a change to the pressure drag. Significantly, a change to the skin friction drag of B2 occurs when placed directly behind towed B1, this dominates the B2's drag reduction. Then at $D / L=$ 4.47 , the total drag of both hulls tends towards the single free stream towed resistance.

For case $\mathrm{F} 2$, at $1.17 \leq D / L \leq 4.47$, the total drag of self-propelled $\mathrm{B} 1$ is increased by $12 \%$ compared to that of towed B1 (F1). The drag of self-propelled B1 tends towards the single vehicle self-propelled thrust as the offset increases. Considering Figure $8 \mathrm{~b}$ and $8 \mathrm{c}$ at $D / L=1.17$, the drag of $\mathrm{B} 2$ is increased by $12 \%$ compared to that of towed B2 (F1), this is due to an addition to the skin friction drag results from a propeller race deduction. As the offset increases, the extra skin friction drag is reduced which results in a drcrease of the total drag augment towards the single towed resistance at $D / L>5.0$.

For case F3, the drag of self-propelled B1 (F3) shows the same results as that of case F2. Apparently, placing either a towed B2 (F2) or self-propelled B2 (F3) directly behind the self-propelled B1 has no impact to the B1's drag. At $D / L=1.17$, the drag of the self-propelled B2 (F3) is increased by $18 \%$ compared to the towed $\mathrm{B} 2(\mathrm{~F} 2)$. The drag of self-propelled B2 (F3) is then reduced towards the free stream thrust when the offset increases. Figure $8 \mathrm{~b}$ and $8 \mathrm{c}$ show that a change of the total drag augment is influenced by the pressure drag rather than the skin friction drag.

For these drafting cases, in general, for vehicles following a self-propelled leader within 5.0L, the skin friction drag of the follower is increased by the propeller race deduction, consequently, a rise in the total drag. At the longitudinal offset $D / L>5.0$, the drags of both $\mathrm{B} 1$ and $\mathrm{B} 2$ tend towards the single free stream value, which are at a towed resistance and a self-propelled thrust for the towed vehicle and self-propelled vehicle, respectively. 
Figure 9 illustrates the influence of the transverse separation in the parallel configuration on the components of drag. Implementing the propeller on both hulls increases the drag on both hulls. For case F1, at $S / L=0.17$, the total drag of both towed vehicles is incresed by $10 \%$, it is due to a change in the pressure drag rather than a skin friction drag. As the separation increases, the drag of both hulls tends towards the drag of single free stream towed resistance.

For cases F2, the total drag of self-propelled B1 is increased by $15 \%$ compared to the towed B1 (F1) and then tends toward the single self-propelled thrust as the separation increases. From Figure $9 \mathrm{~b}$ and $9 \mathrm{c}$, at the separation within $0.27 \mathrm{~L}$, the propeller race decuction results from the self-propelled B1 is driven the pressure drag of towed B2 to be increased by $15 \%$ when compared to the towed B2 (F1), consequently increase a $4 \%$ of its total drag.

For case F3, at $S / L=0.17$, the self-propelled B1 (F3) experiences a $4 \%$ drag augment compared to that of F2. This suggests that the propeller race deduction from both self-propelled AUVs interacts with each other, subsequently disturbing the pressure distribution around the hulls. For $S / L>0.30$, the drag of both self-propelled vehicles tends towards the free stream thrust requirements.

For all drafting and parallel cases, with both vehicles in close proximity, significant change in the pressure drag is dominating an increment in the total drag. The propeller race deduction influences a significant change in the skin friction drag of any vehicle placed directly behind a self-propelled vehicle. On the other hand, at very close proximity in the parallel configuration, the propeller race drives a $25 \%$ increase in the pressure drag for both vehicles, it is evident that a change of the skin friction drag has a minimal change of less than $1 \%$.

\section{Conclusion}

This paper investigates the influence of the propeller race on the upstream and downstream self-propelled AUVs. Simulations are performed for a single hull, two body force models are used to replicate the impact of the propeller by using RANS-HO and RANS-UT to model the momentum source terms. Then, a fleet of twin self-propelled AUVs in a drafting configuration at $D / L=1.47$ is selected as a promising case study. The velocity profile of flow past two drafting AUVs is demonstrated and the mean wake fraction is estimated. The drag of each individual is analysed to determine the hydrodynamic interference due to the propeller.

In drafting at $D / L=1.47$, the results show that the thrust deduction of the self-propelled vehicles is dominated by the propeller's thrust rather than torque. The drag penalty of the leader AUV is due to its own thrust deduction whilst the drag penalty of the follower AUV is significantly increased by both its own thrust deduction and the re-energised flow due to the upstream propeller, named the propeller race deduction.

Using the RANS-UT model, the parametric studies has been extended to investigate the influence of transverse separations and longitudinal offsets on the twin vehicles' fleet. Drafting in close proximity of two self-propelled AUVs, the increase in skin friction drag of the follower is dominated by the propeller race deduction while the increase in the pressure drag is driven by its own thrust deduction. The results of two parallel self-propelled AUVs show the bigger the spacing, the smaller the drag penalty, the towed bare hull drag tends towards the towed resistance at increasing separations, the drag of self-propelled vehicle reduces towards the single vehicle thrust.

By implementing the virtual propeller, the results show no propulsive energy benefit to the individual multiple vehicles in a fleet. However, based on this numerical information, operators can determine the optimal configurations in transverse separation and longitudinal offset based on energy considerations. Since this information is based on the same size and same shape prolate spheroid hulls, by applying the different size and shape hulls in the fleet may impact the body-to-body interaction effect and the fleet drag which may provide positive results for saving energy, especially at the individual level. From a vehicle or mission design perspective, it is important to correctly understand the propulsive energy budget of the vehicle and its impact on both range and endurance. This study highlights the importance of considering both thrust deduction and any propeller race deductions when calculating the propulsive power consumption.

\section{Acknowledgment}

The authors acknowledge the use of the IRIDIS High Performance Computing Facility, and associated support services at the University of Southampton, in the completion of this work. The PhD studentship of Rattanasiri was financed by the Royal Thai Government. 


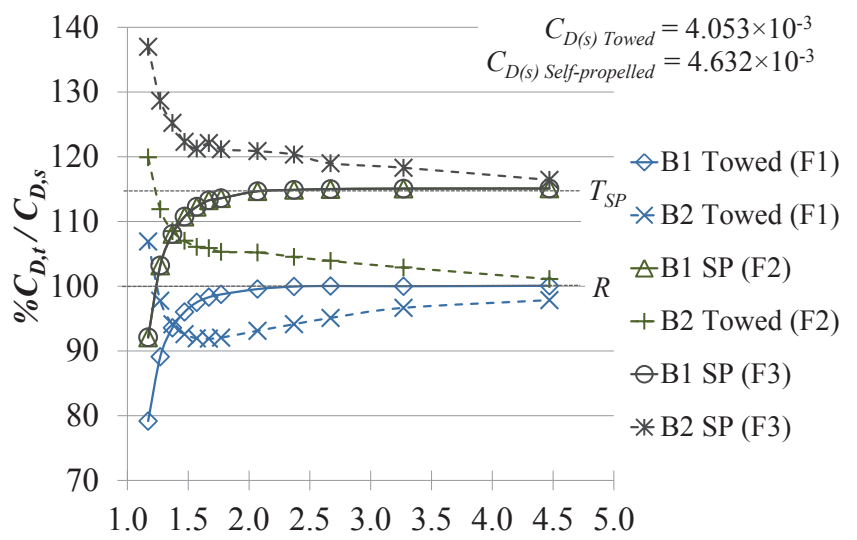

(a) Total drag coefficient

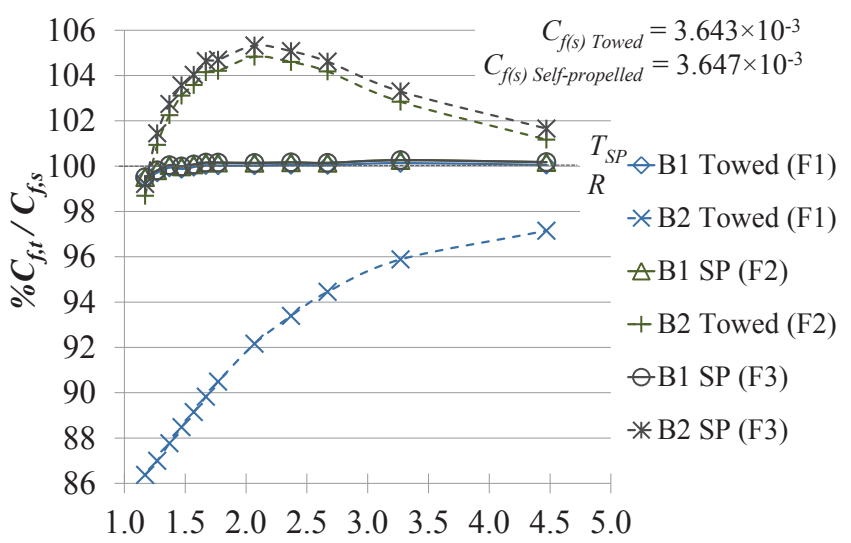

(b) Skin friction drag coefficient

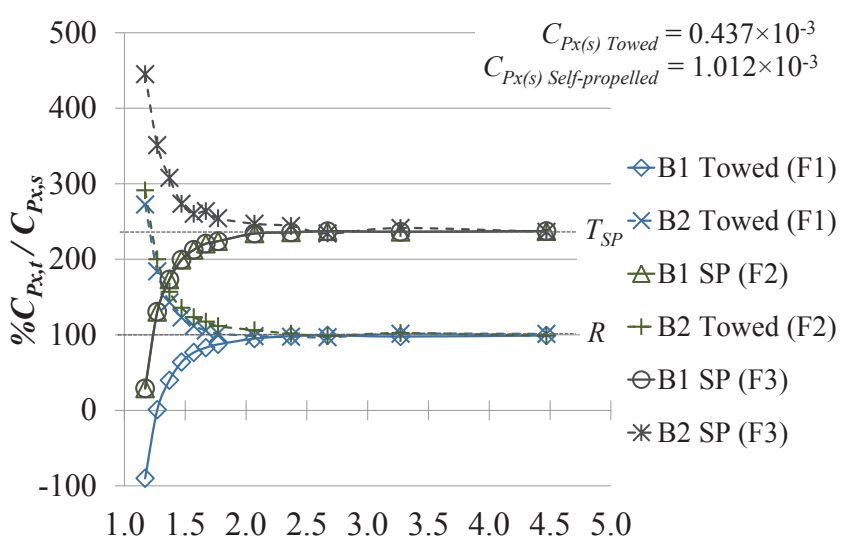

(c) Pressure drag coefficient

Figure 8: Effect of propeller on forces acting on drafting configuration B1 and B2 at various longitudinal offsets 


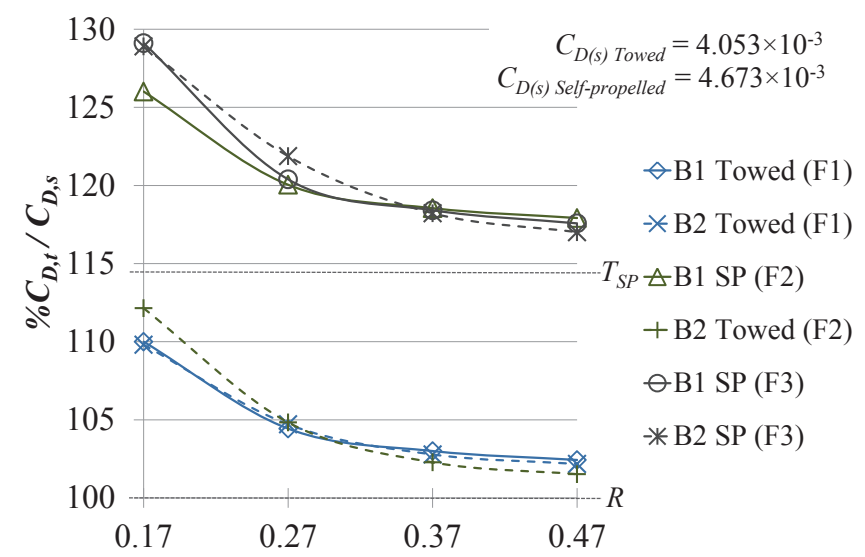

(a) Total drag coefficient

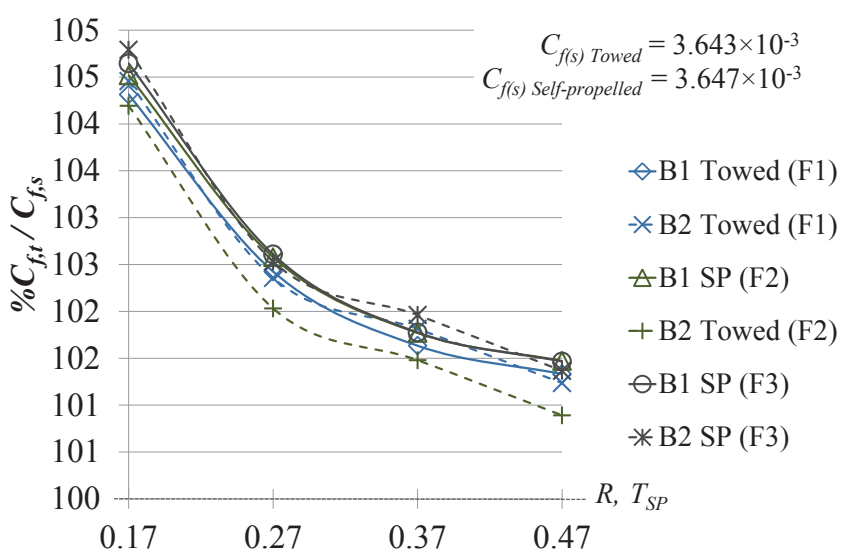

(b) Skin friction drag coefficient

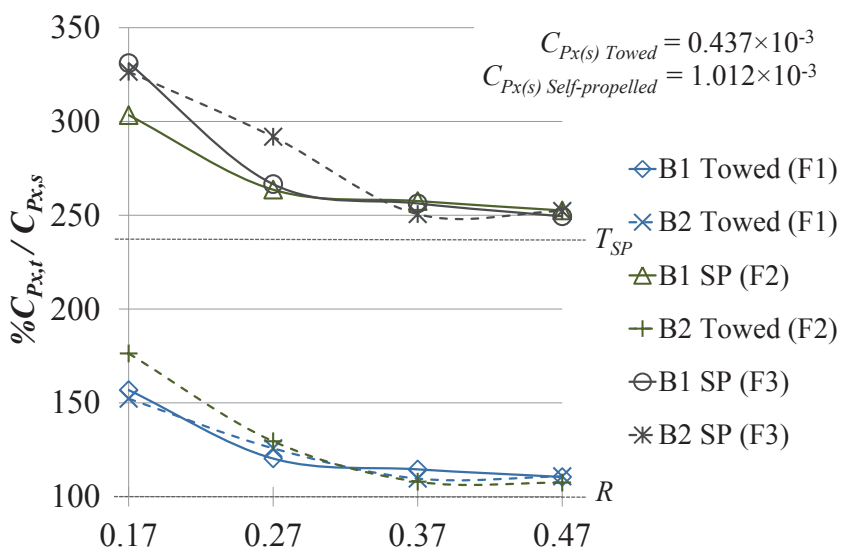

(c) Pressure drag coefficient

Figure 9: Effect of propeller on forces acting on the parallel configuration B1 and B2 at various transverse separations 


\section{References}

Aguiar, A.P., Almeida, J., Bayat, M., Cardeira, B., Cunha, R., Hausler, A., Maurya, P., Oliveira, A., Pascoal, A., Pereira, A., Rufino, M., Sebastiao, L., Silvestre, C., Vanni, F., 2011. Cooperative control of multiple marine vehicles: theoretical challenges and practical issues. Control \& Automation (MED), 2011 19th Mediterranean Conference on , 424-429.

Alexander, R.M., 2004. Hitching a lift hydrodynamically - in swimming, flying and cycling. Journal of Biology 3.

Andersson, M., Wallander, J., 2003. Kin selection and reciprocity in flight formation? Behavioral Ecology 15, $158-162$.

ANSYS, 2010. ANSYS CFX, Release 12.1. ANSYS.

Bean, T., Beidler, G., Canning, J., Odell, D., Wall, R., ORourke, M., Edwards, D., Anderson, M., 2007. AUV cooperative operations using acoustic communication and navigation. IEEE Journal of Oceanic Engineering .

Bellingham, J.G., Willcox, J.S., 1996. Optimizing AUV oceanographic surveys. autonomous underwater vehicle technology., in: AUV '96., Proceedings of the 1996 Symposium on 2-6 Jun 1996.

Botelho, S., Neves, R., Taddei, L., 2005. Localization of a fleet of AUVs using visual maps. OCEANS 2005 Europe 2, 1320-1325.

Bradley, A.M., 1992. Low power navigation and control for long range autonomous underwater vehicles., in: Proceeding of the Second International Offshore and Polar Engineering. Conference, San francisco, CA.

Bradley, A.M., Feezor, M.D., Singh, H., Sorrell, F.Y., 2001. Power systems for autonomous underwater vehicles. IEEE Journal of Oceanic Engineering 26.

Brath, T.J., Jesperson, D.C., 1989. The design and application of upwind schemes on unstructured meshes. AIAA Paper 89-0366.

Burcher, R., Rydill, L., 1994. Concepts in submarine design. Cambridge: Cambridge University Press.

Burger, M., Pavlov, A., Pettersen, K., 2009. Conditional integrators for path following and formation control of marine vessels under constant disturbances., in: The 8th IFAC International Conference on Manoeuvring and Control of Marine Craft, pp. 179-184.

Cui, R., Ge, S.S., How, B.V.E., Choo, Y.S., 2009. Leader-follwer formation control of underactuated AUVs with leader position measurement., in: Proceedings of the 2009 IEEE International Conference on Robotics and Automation, Kobe, Japan, pp. 2441-2446.

Cui, R., Ge, S.S., How, B.V.E., Choo, Y.S., 2010. Leader-follwer formation control of underactuated AUVs. Ocean Engineering, 1491-1502.

Dalton, C., Zedan, M.F., 1980. Design of low-drag axisymmetric shapes by the inverse method. Journal of Hydronautics 15, 48-54.

Edwards, D., Bean, T., Odell, D., Anderson, M., 2004. A leader-follower algorithm for multiple AUV formations., in: Proceedings of IEEE/OES Autonomous Underwater Vehicles, vol. 1 Sebasco, ME, pp. 40-46.

Goldstein, S. (Ed.), 1929. On the vortex theory of screw propellers.. Proc. of the Royal Society (A) 123, 440.

Hanrahan, B., Juanes, F., 2001. Estimating the number of fish in atlantic bluefin tuna schools using models derived from captive school observations. Fishery Bulletin 99(3).

Hoerner, S.F., 1965. Fluid-dynamic drag: practical information on aerodynamic drag and hydrodynamic resistance. (pubished by the author).

Hough, G., Ordway, D., 1965. The generalised actuator disc. Developments in Theor. and Appl. Mech. 2, 317336.

Hucho, W.H., Ahmed, S.R., 1998. Aerodynamics of road vehicles : from fluid mechanics to vehicle engineering. Warrendale, Pa. : Society of Automotive Engineers. 
Huggins, A., Packwood, A.R., 1994. The optimum dimensions for a long-range, autonomous, deep-driving, underwater vehicle for oceanographic research. Ocean Engineering 21, 45-56.

ITTC, 1957. Proceedings of the 8th ITTC, madrid, spain., in: Canal de Experiencias Hidrodinamicas, El Pardo, Madrid.

Jagadeesh, P., Murali, K., 2005. Application of low-re turbulence models for flow simulations past underwater vehicle hull forms. The Journal of Naval Architecture and Marine Engineering 1 (2), 41-54.

Jagadeesh, P., Murali, K., 2006. Investigation of alternative turbulence closure models for axisymmetric underwater hull forms. The Journal of Ocean Technology 1(2), 37-57.

Jagadeesh, P., Murali, M., Idichandy, V.G., 2009. Experimental investigation of hydrodynamic force coefficients over AUV hull form. Journal of Ocean Engineering 36, 113-118.

Karim, M.M., Rahman, M.M., Alim, M.A., 2009. Computation of turbulent viscous flow around submarine hull using unstructured grid. .

Kinsey, J.C., 1998. Drag characterization in the autonomous benthic explorer., in: OCEANS '98 Conference Proceedings 28 Sep-1 Oct 1998, pp. 1696-1700.

Kyle, C.R., 1979. Reduction of wind resistance and power output of recing cyclists and runners traveling in group. Ergonomics 22, 387-397.

Labbe, D.F.L., Wilson, P.A., Weiss, P., Lapierre, L., 2004. Freesub: navigation guidance and control of an IAUV. Royal Institution of Naval Architects Transactions A: International Journal of Maritime Engineering 146(2), 71-9.

Larsson, L., Baba, E., 1996. Advances in marine hydrodynamics. Computational Mechanics Publications. volume 5. chapter Ship resistance and flow computations. pp. 1-75.

Martins, A., Almeida, J.M., Silva, E., 2003. Coordinated maneuver for gradient search using multiple AUVs. OCEANS 2003. Proceedings 1, 247-352.

Menter, F.R., 1994. Two-equation eddy-viscosity turbulence models for engineering applications. AIAA Journal $32(8), 1598-1605$.

Molland, A.F., Turnock, S.R., 1996. A compact computational moethod for peredicting forces on a rudder in a propeller slipstream. In Trans. RINA. 138, 59-71.

Molland, A.F., Utama, I.K.A.P., 1997. Wind tunnel investigation of a pair of ellipsoids in close proximity. Technical Report 98. Ship Science, University of Southampton.

Molland, A.F., Utama, I.K.A.P., 2002. Experimental and numerical investigations of a pair a ellipsoids in close proximity., in: Proceedings of the Institution of Mechanical engineers, Part M: Journal of Engineering for the Maritime Environment.

Parsons, J.S., 1972. The optimum shaping of axisymmetric bodies for minimum drag in incompressible flow. Ph.D. thesis. School of Mechanical Engineering, Purdue University, Lafayette, Ind.

Parsons, J.S., Goodson, R.E., Goldschmied, F.R., 1974. Shaping of axisymmetric bodies for minimum drag in incompressible flow. Journal of Hydronautics 8, 100-107.

Partridge, B.L., Johansson, J., Kalish, J., 1983. The structure of schools of giant bluefin tuna in cape cad bay. Environmental Biology of Fishes 9, 253-262.

Paterson, E.G., Wilson, R.V., Stern, F., 2003. General purpose parallel unsteady RANS ship hydrodynamics code CFDSHIP-IOWA. IIHR Rep.. Univ. of Iowa.

Phillips, A., Turnock, S., Furlong, M., 2008. Comparisons of CFD simulations and in-service data for the self propelled performance of an Autonomous Underwater Vehicle., in: 27th Symposium of Naval Hydrodynamics, Seoul, Korea, 05 - 10 Oct 2008.

Phillips, A., Turnock, S., Furlong, M., 2009a. Simulation of a self-propelled ship using a blade element momentum propeller model and rans. Ocean Engineering 36, 1217-1225. 
Phillips, A., Turnock, S.R., Furlong, M., 2010a. Accurate capture of propeller-rudder interaction using a coupled blade element momentum-rans approach. Ship Technology Research. Schiffstechnik. 57.

Phillips, A.B., 2009. Cost effective hydrodynamic concept design of autonomous underwater vehicle. Ph.D. thesis. School of Engineering Sciences.

Phillips, A.B., Furlong, M., Turnock, S.R., 2007. The use of computational fluid dynamics to assess the hull resistance of concept autonomous underwater vehicles., in: OCEANS 2007 - Europe. Richardson TX, USA, Institute of Electrical and Electronics Engineers.

Phillips, A.B., Turnock, S.R., Furlong, M., 2010b. Influence of turbulence closure models on the vortical flow field around a submarine body undergoing steady drift. Journal of Marine Science and Technology 15(3), $201-217$.

Phillips, A.B., Turnock, S.R., Furlong, M., 2010c. The use of computational fluid dynamics to aid cost-effective hydrodynamic design of autonomous underwater vehicles., in: Proceedings of the Institution of Mechanical Engineers, Part M: Journal of Engineering for the Maritime Environment, pp. 239-254.

Phillips, A.B., Turnock, S.R., Furlong, M.E., 2009b. Evaluation of manoeuvring coefficients of a self-propelled ship using a blade element momentum propeller model coupled to a reynolds averaged navier stokes flow solver. Ocean Engineering 36, 1217-1225.

Rattanasiri, P., Wilson, P.A., Phillips, A.B., 2013. Numerical investigation of a fleets of towed auvs. Submitted (Under review).

Reeder, C.A., Odell, D.L., Okamoto, A., Anderson, M.J., Edwards, D.B., 2004. Two-hydrophone heading and range sensor applied to formation-flying for AUVs., in: OCEANS '04. MTTS/IEEE TECHNO-OCEAN '04, pp. 517-523.

Rhie, C.M., Chow, W.L., 1982. A numerical study of the turbulent flow past an isolated airfoil with trailing edge separation. AIAA Paper 82-0998.

Sarkar, T., Sayer, P.G., Fraser, S.M., 1997a. Flow simulation past axisymmetric bodies using four different turbulence model. Application Mathematic Modelling 21, 783-792.

Sarkar, T., Sayer, P.G., Fraser, S.M., 1997b. A study of autonomous underwater vehicle hull forms using computational fluid dynamics. International journal for Numerical Methods in Fluids 25, 1301-1313.

Silva, A.J., Rouboa, A., Moreira, A., Reis, V.M., Alves, F., Vilas-Boas, J.P., Marinho, D.A., 2008. Analysis of drafting effects in swimming using computational fluid dynamics. Journal of Sports Science and Medicine 7 , 60-66.

Stern, F., Kim, H.T., Cheng, H.C., 1988. A viscous-flow approach to the computation of of propeller-hull interaction. Journal Ship Research 32, 246-262.

Stevenson, P., Furlong, M., Dormer, D., 2007. AUV shapes - combining the practical and hydrodynamic considerations., in: OCEANS 2007 - Europe.

Turnock, S.R., Phillips, A.B., Furlong, M., 2008. URANS Simulations of statics drift and dynamic manoeuvres of the KVLCC2 tanker., in: In SIMMAN Int. Manoeuvring Workshop, Copenhagen.

Vanni, F.V., 2007. Coordinated motion control of multiple autonomous under vehicle. Master's thesis. Technical University of Lisbon.

Weihs, D., 2004. The hydrodynamics of dolphin drafting. Journal of Biology 3:8.

Weihs, D., Ringel, M., Victor, M., 2007. Aerodynamic interactions between adjacent slender bodies instrumentation and wind tunnel testing. Instrumentation in Aerospace Simulation Facilities, 2007. ICIASF 2007. 22nd International Congress on , 1-28.

Weiss, P., Mascarelli, J.V.C., Grossnet, D., Brignone, L., Labbe, D.F.L., Wilson, P.A., 2003. FREESUB: Dynamic stabilization and docking for autonomous underwater vehicles., in: The 13th International Symposium on Unmanned Untethered Submersible Technology, Durham, USA. 


\section{Nomeclature}

\begin{tabular}{|c|c|}
\hline $1+k$ & The form factor of a single spheroid \\
\hline $1+\beta k$ & The form factor of twin spheroids \\
\hline$A_{w}$ & Wetted surface area $\left(\mathrm{m}^{2}\right)$ \\
\hline $\mathrm{B} 1$ and $\mathrm{B} 2$ & Leading spheroid and following spheroid \\
\hline$\% B 1$ and $\% B 2$ & Individual drag of $\mathrm{B} 1$ and $\mathrm{B} 2$ referred to a single hull drag \\
\hline$\% C B$ & Combined drag refer to sum of two single hull drags \\
\hline$C_{D}$ & Total drag coefficient, $\frac{\text { Total drag }}{0.5 \rho V^{2} A_{w}}$ \\
\hline$C_{D(\mathrm{~B} 1)}$ and $C_{D(\mathrm{~B} 2)}$ & Drag coefficient of B1 and B2 in fleet \\
\hline$C_{D(s)}$ & Drag coefficient of a single hull \\
\hline$C_{F}$ & Skin friction drag coefficient, $\frac{\text { Skin friction drag }}{0.5 \rho V^{2}}$ \\
\hline$\left(C_{F}\right)_{1957}$ & Skin friction drag coefficient proposed by (ITTC, 1957) \\
\hline$C_{P x}$ & Pressure drag coefficient, $\frac{\text { Pressure drag }}{0.5 \rho V^{2}}$ \\
\hline$C_{S F}$ & Coefficient of side-force \\
\hline$C_{t h}$ and $K_{T}$ & Thrust coefficient \\
\hline$d_{m}$ & Maximum diameter of the body of revolution (m) \\
\hline$D$ & Longitudinal offset (m) \\
\hline$D / L$ & Non-dimensional longitudinal offset \\
\hline$d_{h}$ & Maximum hull diameter (m) \\
\hline$F \bar{b}_{x}$ & Non-dimensional axial momentum source term \\
\hline$F \bar{b}_{\theta}$ & Non-dimensional tangential momentum source term \\
\hline$F \bar{b}_{r}$ & Non-dimensional radial momentum source term \\
\hline$J$ & Advance coefficient \\
\hline$K_{Q}$ & Torque coefficient \\
\hline$n$ & Revolutions per second of propeller $\left(\mathrm{s}^{-1}\right)$ \\
\hline$S / L$ & Transverse separation \\
\hline$t$ & Thrust deduction \\
\hline$T_{D}$ & The self-propelled drag $(\mathrm{N})$ \\
\hline$T_{S P}$ & The require thrust $(\mathrm{N})$ \\
\hline$L$ & Length of the body from nose to tail (m) \\
\hline$R$ & Towed drag $(\mathrm{N})$ \\
\hline $\operatorname{Re}$ & Length Reynolds number, $\frac{V L}{v}$ \\
\hline$R_{p}$ and $R_{h}$ & Radius of propeller and hub (m) \\
\hline$r_{p}$ & Local propeller radius $(\mathrm{m})$ \\
\hline$V$ & Vehicle speed $(\mathrm{m} / \mathrm{s})$ \\
\hline$V_{a}$ & Advance speed $(\mathrm{m} / \mathrm{s})$ \\
\hline$U_{i}$ & Cartesian mean velocity components $\left(\overline{U_{x}}, \overline{U_{y}}, \overline{U_{z}}\right)$ \\
\hline$w_{t}$ & Wake fraction \\
\hline $\bar{w}_{t}$ & mMean wake fraction over propeller disc \\
\hline$x_{i}$ & Represents Cartesian co-ordinates $(X, Y, Z)(\mathrm{m})$ \\
\hline$v$ & Fluid kinematic viscosity, $\mu / \rho\left(\mathrm{m}^{2} / \mathrm{s}\right)$ \\
\hline$\rho$ & Fluid density $\left(\mathrm{kg} / \mathrm{m}^{3}\right)$ \\
\hline$\mu$ & Fluid dynamic viscosity (kg/m.s) \\
\hline$\mu_{t}$ & Turbulent viscosity $(\mathrm{kg} / \mathrm{m} . \mathrm{s})$ \\
\hline$k$ & Fluid turbulent kinetic energy $\left(\mathrm{m}^{2} / \mathrm{s}^{2}\right)$ \\
\hline$\epsilon$ & Rate of dissipation of turbulent energy $\left(\mathrm{m}^{2} s^{-3}\right)$ \\
\hline$\widetilde{v}$ & Viscosity like variable \\
\hline$\gamma$ & The propeller race deduction \\
\hline$\Delta$ & The difference between drags \\
\hline
\end{tabular}

